\title{
Direct Observation of the Strong Interaction Between Carbon Nanotubes and Quartz Substrate
}

\author{
Lei Ding ${ }^{1}$, Weiwei Zhou ${ }^{1}$, Thomas P. McNicholas ${ }^{1}$, Jinyong Wang ${ }^{2}$, Haibin $\mathrm{Chu}^{2}$, Yan $\mathrm{Li}^{2}$, and Jie Liu ${ }^{1}(\bowtie)$ \\ ${ }^{1}$ Department of Chemistry, Duke University, Durham, North Carolina 27708, USA \\ ${ }^{2}$ Beijing National Laboratory for Molecular Sciences, Key Laboratory for the Physics and Chemistry of Nanodevices, National \\ Laboratory of Rare Earth Material Chemistry and Application, College of Chemistry and Molecular Engineering, Peking University, \\ Beijing 100871, China
}

Received: 9 September 2009 / Revised: 8 October 2009 / Accepted: 11 October 2009

(CTsinghua University Press and Springer-Verlag 2009. This article is published with open access at Springerlink.com

\begin{abstract}
We present a chemical vapor deposition (CVD) method for the growth of uniform single-walled carbon nanotube (SWNT) arrays on a stable temperature (ST)-cut single crystal quartz substrate using a mixture of methanol and ethanol as carbon source. It is found that introducing methanol during the growth can improve the density and the length of the well-aligned SWNTs in the arrays as well as increase the SWNT/quartz interaction. Obvious "up-shifts" of G-band frequencies in the Raman spectra have been found for the aligned SWNTs. A welldesigned control experiment shows that the G-band "up-shifts" originate from the strong interaction between SWNTs and the quartz substrate. It is believed that exploring this interaction will help to elucidate the growth mechanism; ultimately, this will help realize the promise of controlling the chirality of SWNTs.
\end{abstract}

\section{KEYWORDS}

Single-walled carbon nanotube, quartz wafer, Raman spectroscopy, G-band "up-shift", chemical vapor deposition (CVD)

\section{Introduction}

Recently, horizontally aligned single-walled carbon nanotube (SWNT) arrays have been heavily studied for their promise in potential applications, including high-frequency transistors [1], radio frequency analog electronics [2], field effect transistors [3], and polarization-sensitive near-field probes [4]. Compared with other approaches such as selfassembly [5], electrospinning [6], and direct growth approaches with the assistance of an electric field [7] and feed gas flow $[8,9]$, the surface lattice-guided growth of SWNTs on single crystal substrates has been an important advancement because of the high density well-aligned nanotube samples it produces [10-16].

It is believed that the nanotube/substrate interaction, which involves the nanotube/lattice or nanotube/atomic steps interaction, plays an important role in the observed nanotube alignment on single-crystal substrates [11, 12]. Our recent work also shows that the nanotube/substrate interaction is crucial and necessary for the growth of a significantly enhanced fraction of semiconducting SWNTs on

Address correspondence to j.liu@duke.edu 
ST-cut quartz [17]. But until now, little work has been done for investigating the mechanism of the nanotube/substrate interaction. Most of the work that has been reported is based on molecular simulation, as direct proof is lacking. One possible reason is that the nanotube/substrate interaction has little influence on the electrical and spectral properties of SWNTs, which make the direct characterization and observation of this interaction difficult.

In this article, we present a method to observe the strong interaction between the nanotubes and a quartz lattice. In our experiments, a high ratio of methanol: ethanol was used as the carbon feed gas for the growth of high-density ( 20 SWNTs / $\mu \mathrm{m})$ and long $(\sim 1 \mathrm{~mm})$ SWNT arrays on stable temperature (ST)-cut single crystal quartz substrates. Raman measurements were used to characterize the interaction between the aligned SWNTs and the quartz surface. Raman characterization of the samples shows that the strong interaction can cause obvious G-band "up-shift" of the SWNTs from $1590 \mathrm{~cm}^{-1}$ to around $1610 \mathrm{~cm}^{-1}$. It is believed that this strong interaction originates from the matching of $\mathrm{C}$ atoms in the sidewall of the SWNTs with the $\mathrm{Si}$ and $\mathrm{O}$ atoms in the quartz lattice [12]; this may also play an important role in the selective growth of semiconducting nanotubes reported earlier [17].

\section{Experimental}

The substrates were ST-cut single-crystal quartz wafers ( $36^{\circ}$ Y-cut) obtained from Hoffman Materials Inc. and used without any further treatment. 200 proof $(99.5 \%)$ ethanol was purchased from Fisher Scientific and used as the catalyst precursor solvent and as carbon precursor. $99.9 \%$ pure methanol was purchased from Fisher Scientific and used as the other component in the carbon precursor. Polyvinylpyrrolidone (PVP) with an average $M_{\mathrm{w}}$ ca. 55000 and $99 \%$ pure anhydrous $\mathrm{CuCl}_{2}$ were purchased from Aldrich Chemical Company, Inc. and Acros Organics, respectively. $\mathrm{A} \mathrm{CuCl}_{2}(1.0$ $\mathrm{mmol} / \mathrm{L}) / \mathrm{PVP}$ (10 $\mathrm{mmol} / \mathrm{L})$ alcohol solution was used to deposit catalyst onto the substrate. The growth experiments were performed in a 1-inch tube furnace at $900{ }^{\circ} \mathrm{C}$. The catalyst patterning and growth processes were similar to the method reported previously [17]. Typically, the substrate with catalyst precursor deposited on it was annealed in air at $750{ }^{\circ} \mathrm{C}$ for $10 \mathrm{~min}$ to remove the PVP in the catalyst precursor. Then, the substrate was heated to $800{ }^{\circ} \mathrm{C}$ and kept for $15 \mathrm{~min}$ in a flow of hydrogen (750 standard cubic centimeters per minute (sccm)), followed by chemical vapor deposition (CVD) growth of SWNTs at $900{ }^{\circ} \mathrm{C}$ in a flow of hydrogen (750 sccm) and argon (600 sccm, through a methanol bubbler and $150 \mathrm{sccm}$, through an ethanol bubbler). After 15 min growth, the sample was cooled to room temperature and inspected by scanning electron microscopy (SEM), atomic force microscopy (AFM), and Raman spectrometry.

The transfer process of the SWNTs from quartz to $\mathrm{Si}, \mathrm{SiO}_{x} / \mathrm{Si}$ and quartz wafers was similar to the method reported previously [17]. Briefly, a drop of 8\% poly(methyl methacrylate) (PMMA) solution (A4) was spin casted on SWNT/quartz to form a PMMA layer. After this, the PMMA/SWNT/quartz substrate was baked at $160{ }^{\circ} \mathrm{C}$ for $10 \mathrm{~min}$ and immersed in boiling $\mathrm{KOH}(1 \mathrm{~mol} / \mathrm{L})$ aqueous solution for $40 \mathrm{~min}$. The PMMA/SWNTs film was then peeled off from the substrate. Then, the PMMA/SWNTs film was washed with nano-pure water and carefully picked up with the other substrates. The angle between the aligned SWNTs and the [100] lattice direction on a quartz substrate can be controlled accurately in the pick-up process. Lastly, the PMMA/SWNTs/ substrate was baked at $160{ }^{\circ} \mathrm{C}$ for $10 \mathrm{~min}$ and then washed with acetone and water. The SWNTs transferred onto the trenched quartz were parallel to the [100] direction of the lattice. In contrast to other methods [18], our transfer method is metal-free. The SWNTs transferred onto other substrates were shown to be clean and well aligned.

SEM images were taken on an FEI XL30 SEM-FEG instrument with $1-\mathrm{kV}$ acceleration voltage. AFM images were taking on a Nanoscope IIIa instrument from Vecco Inc. in the tapping mode ${ }^{\mathrm{TM}}$. The Raman instrument used was a LabRam ARAMIS from Horiba Jobin Yvon with an excitation laser line of $633 \mathrm{~nm}$. The excitation radiation was polarized parallel to the aligned SWNTs during the Raman measurements. 


\section{Results and discussion}

\subsection{Growth of SWNT arrays with a high ratio of methanol:ethanol}

Our previous results showed that perfectly aligned SWNT arrays consisting almost exclusively of semiconducting nanotubes can be grown on ST-cut single crystal quartz substrates [17]. The density of the aligned SWNTs in the arrays was $\sim 5$ SWNTs $/ \mu \mathrm{m}$. It was observed that the introduction of methanol can enhance the ratio of semiconducting SWNTs in the arrays. Herein, we present an improved CVD method using a higher ratio of methanol:ethanol (600 sccm through a methanol bubbler and $150 \mathrm{sccm}$ through an ethanol bubbler) as carbon feed gas during growth. Using these conditions, it was found that higher-density and uniform SWNT arrays can be grown on ST-cut single crystal quartz substrates. Figure 1 displays the SEM and AFM images of SWNT arrays grown on the ST-cut quartz. The nanotubes are distributed uniformly along the [100] direction over the whole surface with a density as high as 20 SWNTs / $\mu \mathrm{m}$. The density of the aligned SWNTs is so high that it is difficult to distinguish the isolated
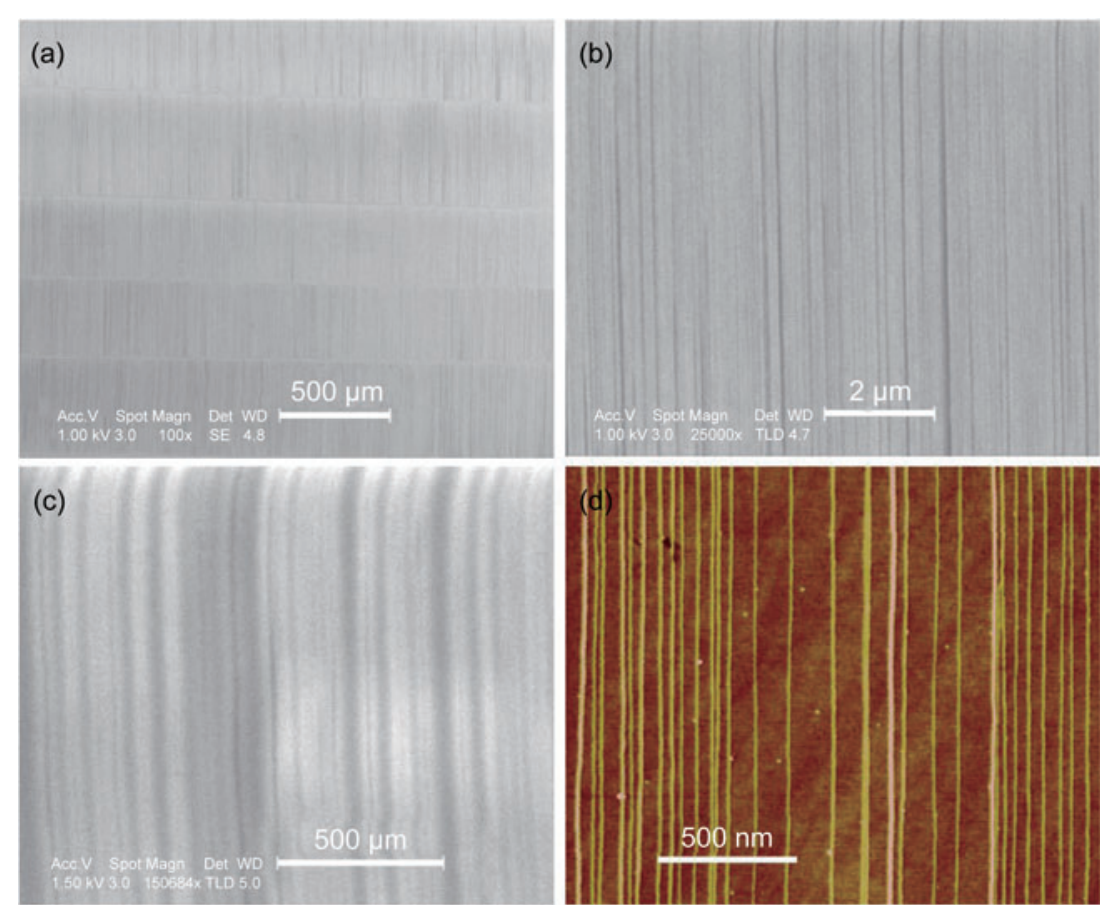

Figure 1 Arrays of high density SWNTS grown on ST-cut single crystal quartz with a large ratio of methanol:ethanol used as carbon precursors: (a)-(c) SEM images; the bright and parallel horizontal lines visible in (a) are catalyst lines; (d) AFM image
SWNTs on the surface when the magnification of the SEM images is below 150000X. A possible reason for the increased density could be the $\mathrm{OH}$ radicals coming from methanol, which can etch amorphous carbon and keep the catalyst nanoparticles from being poisoned. This would result in more catalyst nanoparticles being active during growth. Such a high-density and uniform SWNT array can be used directly as an anisotropic thin-film conductor.

Also, we found that the average length of the SWNTs grown with a high ratio of methanol:ethanol can be of the order of mm. Earlier research showed that the aligned SWNTs grown from methane or ethanol using $\mathrm{Fe}$ as catalyst have an average length of $\sim 200 \mu \mathrm{m}$ [12]. This work shows that the length of the SWNTs can be improved dramatically using a higher ratio of methanol:ethanol as carbon precursors. Figures 2(a) and 2(b) show SEM images of the arrays of SWNTs on ST-cut quartz. The uniform arrays of long SWNTs can be found on a large scale. The density of the SWNTs near the catalyst is between 5 and 25 SWNTs/ $\mu \mathrm{m}$. In the center of the array marked by the dotted rectangle in Fig. 2(a) the average density is still as high as $2.2 \mathrm{SWNTs} / \mu \mathrm{m}$ (see Electronic Supplementary Material (ESM), Fig. S-1). Some of the SWNTs in the array are as long as $2 \mathrm{~mm}$. Figure 2(c) shows the length distribution of the aligned SWNTs in the arrays. Most of the nanotubes have approximate lengths $\geq 1 \mathrm{~mm}$. Our earlier results show that the density of the SWNTs grown from ethanol can be as high as $22 \mathrm{SWNTs} / \mu \mathrm{m}$, but the average length is only about $500 \mu \mathrm{m}$ [14]. So we believe the higher density and longer length of the nanotubes grown here originate from the high ratio of methanol introduced into the growth process. We believe that, at high temperatures, the $\mathrm{OH}$ radicals from methanol can etch amorphous carbon on the catalyst and thus keep it active during SWNT growth [19]. Therefore, the length of the SWNT can be increased by these long-lived 

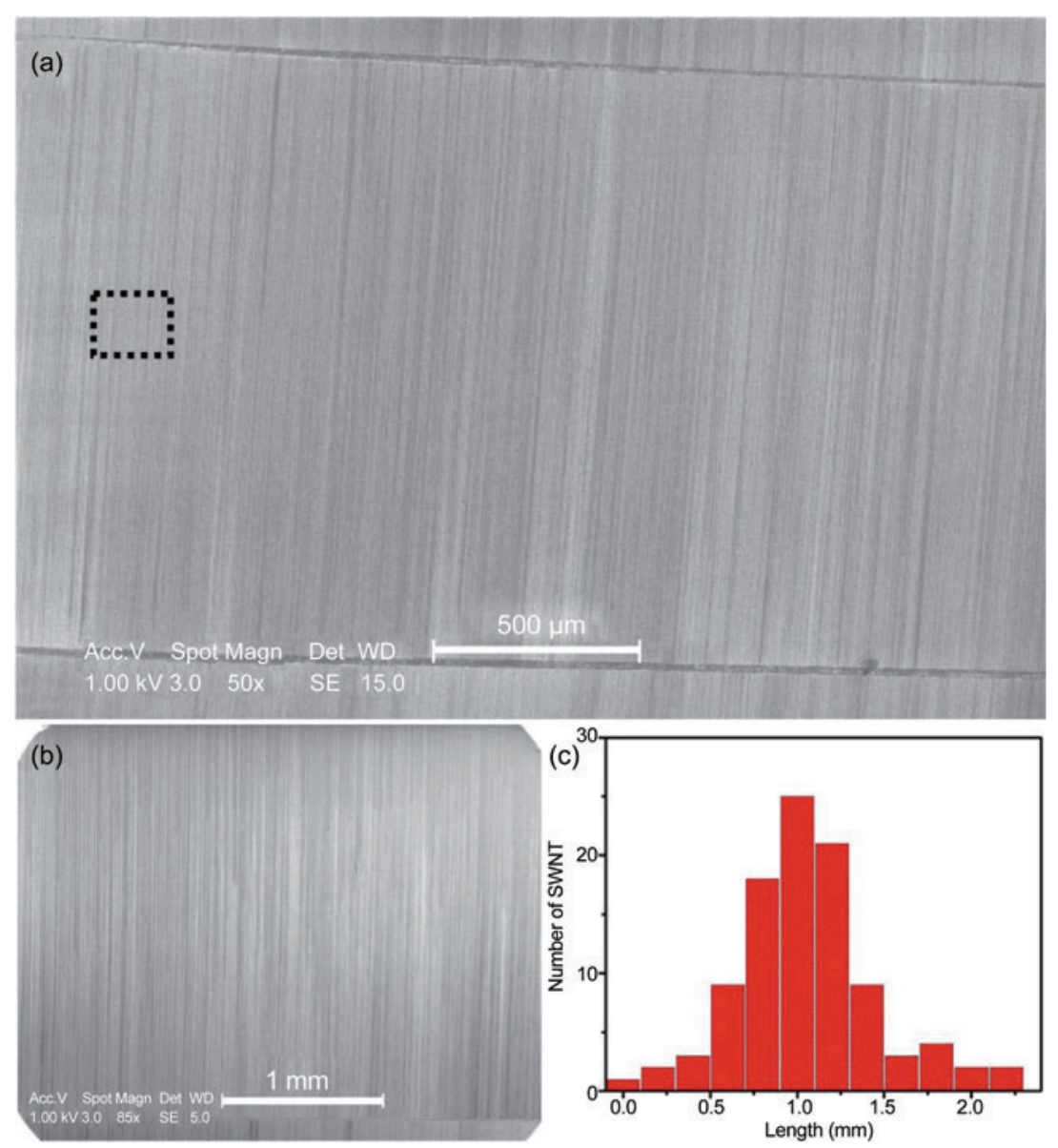

Figure 2 Arrays of long SWNTs grown on ST-cut single crystal quartz with a large ratio of methanol:ethanol used as carbon precursors: (a) and (b) SEM images; the black and parallel horizontal lines visible in (a) are two catalyst lines; the catalyst line in (b) is at the bottom of the SEM; (c) is the length distribution of the SWNTs in the arrays catalyst nanoparticles. However, it should be noted that the length of the SWNT from surface-guided growth cannot be as long as that from flowdirected growth. Surface defects, unreacted nanoparticles, and other SWNTs can all terminate the growth of aligned SWNTs grown on surfaces. Nevertheless, the fabrication of $\mathrm{mm}$ long SWNT arrays can still expand the potential applications of aligned SWNTs to include antennas [20].

\subsection{Resonant Raman spectroscopy (RRS) characterization}

RRS has been proven to be a powerful tool for revealing the remarkable structure and the unusual electronic and phonon properties of SWNTs [21]. Here, RRS has been used to characterize the SWNT arrays grown on ST-cut quartz using a high ratio of methanol:ethanol as carbon precursors. The spectra in Fig. 3 were obtained using a 633-nm excitation laser line at 10 different spots over the substrate. Figure 3(a) shows the radial

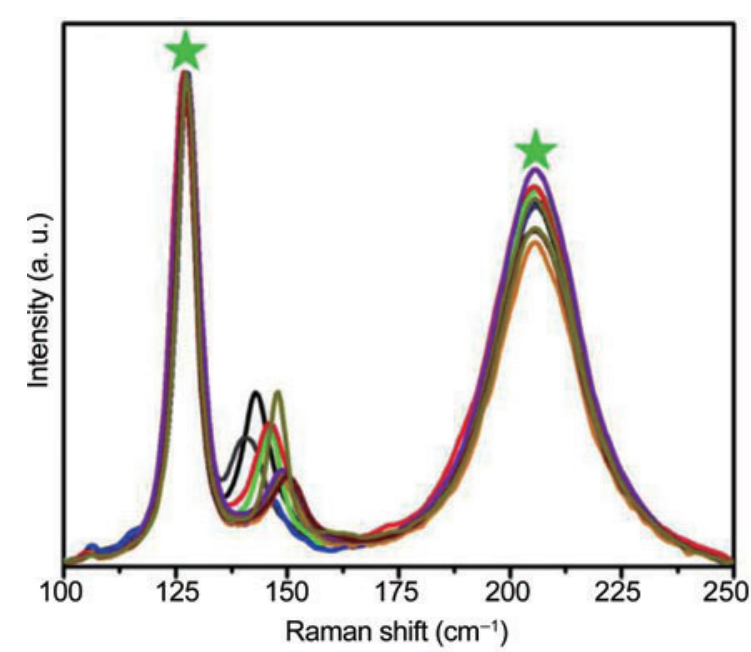

(a)

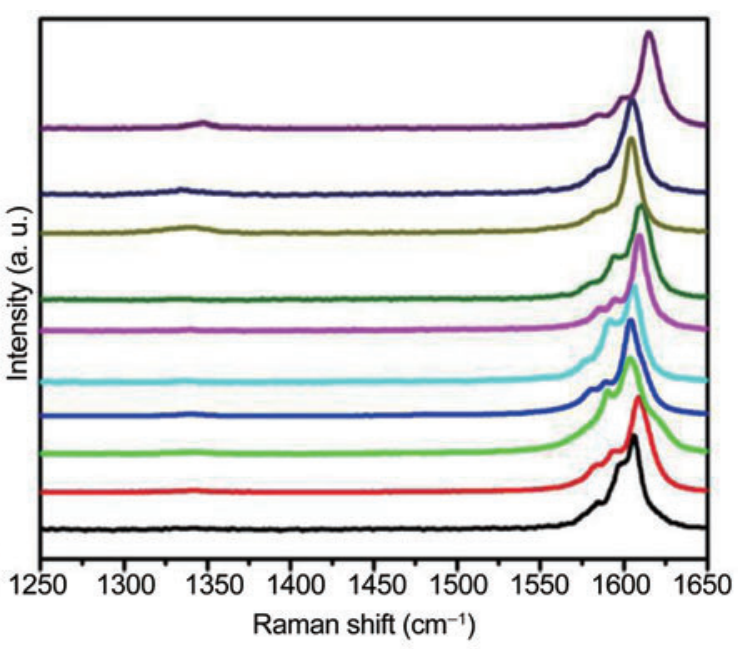

(b)

Figure 3 Raman spectra of the SWNTs grown on ST-cut single crystal quartz with a large ratio of methanol:ethanol used as carbon precursors: (a) RBM spectra; (b) G-band spectra. The Raman spectra were recorded with an excitation laser line of $633 \mathrm{~nm}$. Ten different spots of the sample were characterized over the entire surface. Each curve in a panel shows a spectrum at a different spot on the substrate. The sharp peaks at $127 \mathrm{~cm}^{-1}$ and the broad peaks at $205 \mathrm{~cm}^{-1}$, marked with green stars in (a), correspond to the Raman spectra of the quartz 
breathing mode (RBM) of the as-grown SWNT arrays on the quartz substrate. A narrow distribution of RBM frequencies between $143 \mathrm{~cm}^{-1}$ and $151 \mathrm{~cm}^{-1}$ can be found in Fig. 3(a); these RBM peaks correspond to semiconducting nanotubes as predicted from the socalled Kataura plot [21]. The observation is in good agreement with our earlier results [17]. Figure 3(b) shows that the G-band features of the SWNTs in the arrays on quartz. Interestingly, obvious "up-shifts" of the G-bands with $\mathrm{G}^{+}$frequencies between $1605 \mathrm{~cm}^{-1}$ and $1615 \mathrm{~cm}^{-1}$ can be found in the spectra. No upshifts of G-bands were found on SWNT samples grown on Si wafers with thermal oxides side by side with the quartz substrates. This indicates that the strong SWNT/quartz interaction is likely the main reason for this observed up-shift.

In order to study the mechanism responsible for the up-shifts, we transferred the aligned SWNT arrays onto $\mathrm{SiO}_{x}$ wafers and characterized them by
RRS. Figures 4(a) and 4(b) present the SEM images of the aligned SWNTs transferred onto $\mathrm{SiO}_{x}$ wafers. The alignment of the SWNTs in the arrays is still perfect and essentially no changes were found after the transfer process. Figure 4(c) shows the RBMs of the aligned SWNTs on 10 different spots of the $\mathrm{SiO}_{x}$ substrate. The distribution of the RBM frequencies is between $143 \mathrm{~cm}^{-1}$ and $156 \mathrm{~cm}^{-1}$, which is similar to that shown in Fig. 3(a). Interestingly, as shown in Fig. 4(d), the aligned SWNTs transferred onto $\mathrm{SiO}_{x}$ demonstrate G-band frequencies between $1589 \mathrm{~cm}^{-1}$ and $1593 \mathrm{~cm}^{-1}$ as is observed from SWNTs directly grown on $\mathrm{SiO}_{x}$ surfaces. It is obvious that the "upshift" of the G-bands disappeared after the SWNTs were transferred onto the $\mathrm{SiO}_{x}$ substrate. The same phenomenon was found when the aligned SWNTs were transferred onto a Si substrate. Therefore, we have confirmed that the "up-shift" of the G-band frequencies originates from the strong interaction
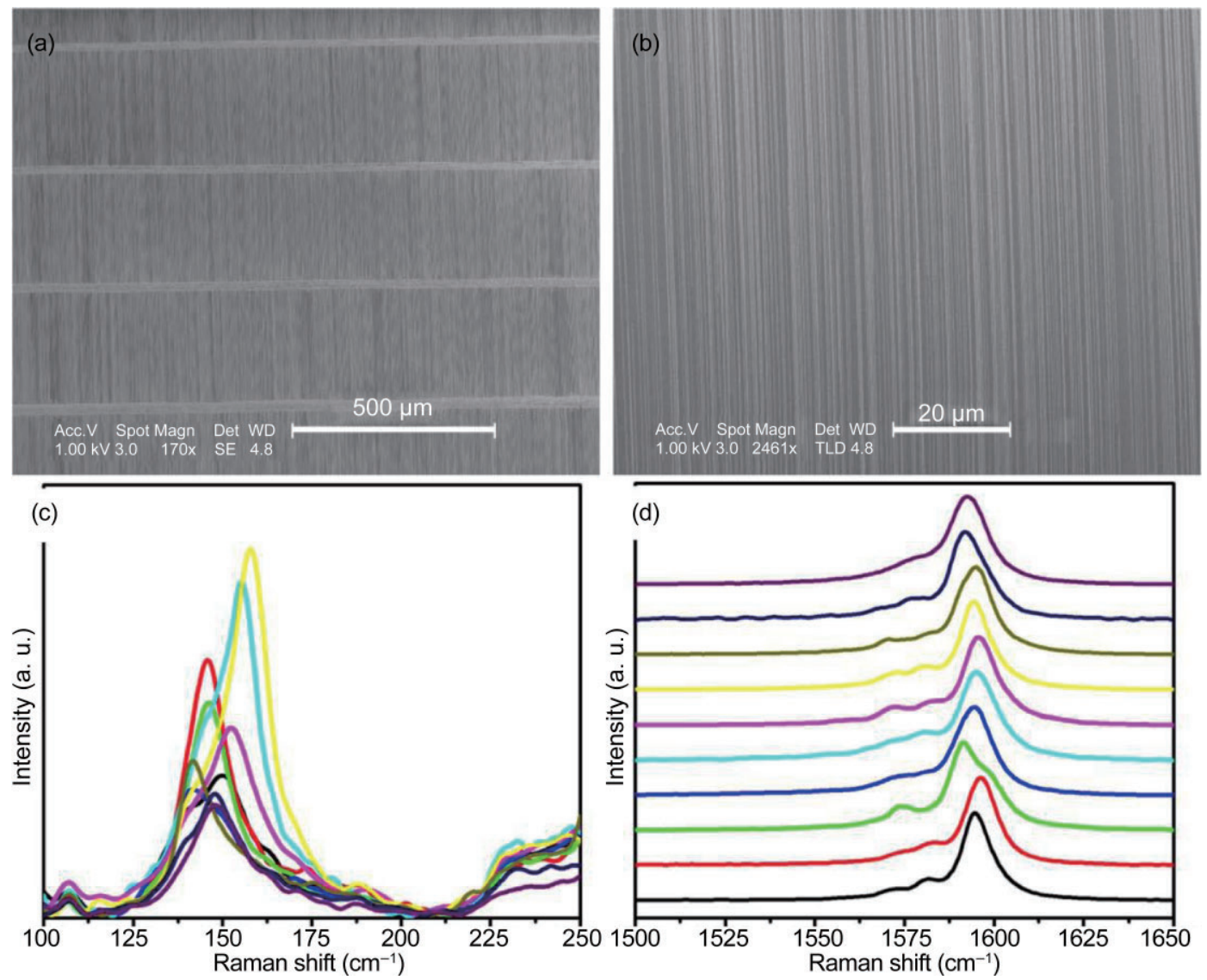

Figure 4 Arrays of aligned SWNTs transferred onto the $\mathrm{SiO}_{x}$ substrate: (a) and (b) SEM images; (c) RBM spectra; (d) G-band spectra. The Raman spectra were recorded with an excitation laser line of $633 \mathrm{~nm}$. Ten different spots of the sample were characterized over the entire surface. Each curve in a panel shows a spectrum at a different spot on the substrate 
between the SWNTs and quartz substrate. Once the aligned SWNTs had been transferred onto the $\mathrm{SiO}_{x}$ substrate, the strong interaction between SWNTs and quartz disappeared and the aligned SWNTs demonstrated G-bands around $1590 \mathrm{~cm}^{-1}$ as they usually do.

Another experiment was performed to further confirm this assertion. In this experiment, we transferred the as-grown SWNTs across a trench etched into an ST-cut single crystal quartz wafer. The orientation of the aligned SWNTs transferred onto the trenched quartz is parallel with the [100] direction of the lattice as shown in Fig. 5. After transfer RRS was used to characterize the portions of the nanotubes in contact with the quartz (positions 1, 3, 5 in Fig. 5) as well as the parts suspended over the trench (positions 2, 4 in Fig. 5) of the same SWNT. Each of the inserted spectra shows the RBM of the SWNT measured simultaneously with the G-band. As shown in Fig. 5, the inserted spectra of RBMs demonstrate frequencies between $152 \mathrm{~cm}^{-1}$ and $153 \mathrm{~cm}^{-1}$, and no significant difference was found between the contacted parts and the suspended parts. But the G-bands of the contacted parts, which show frequencies at $1605 \mathrm{~cm}^{-1}$, $1605 \mathrm{~cm}^{-1}$, and $1603 \mathrm{~cm}^{-1}$, are very different from those of the suspended parts which demonstrate frequencies at $1591 \mathrm{~cm}^{-1}$. This experiment further confirmed the existence of the strong interaction between the aligned SWNTs and the quartz substrate. Other orientations $\left(90^{\circ}\right.$ and $45^{\circ}$ angles with the [100] direction) of the transferred SWNTs on quartz have also been studied. The obvious "up-shifts" of G-bands can still be found on SWNT/quartz, although the frequencies are a little lower than those from the SWNTs parallel with the [100] direction. More SEM images and Raman spectra can be found in Fig. S-2 in the ESM.

Although the mechanism of the G-band "upshifts" caused by the affinity interaction is not fully understood, we present the following hypothesis. The G-band of SWNTs consists of two components, one centered at $1590 \mathrm{~cm}^{-1}\left(\mathrm{G}^{+}\right.$, associated with carbon
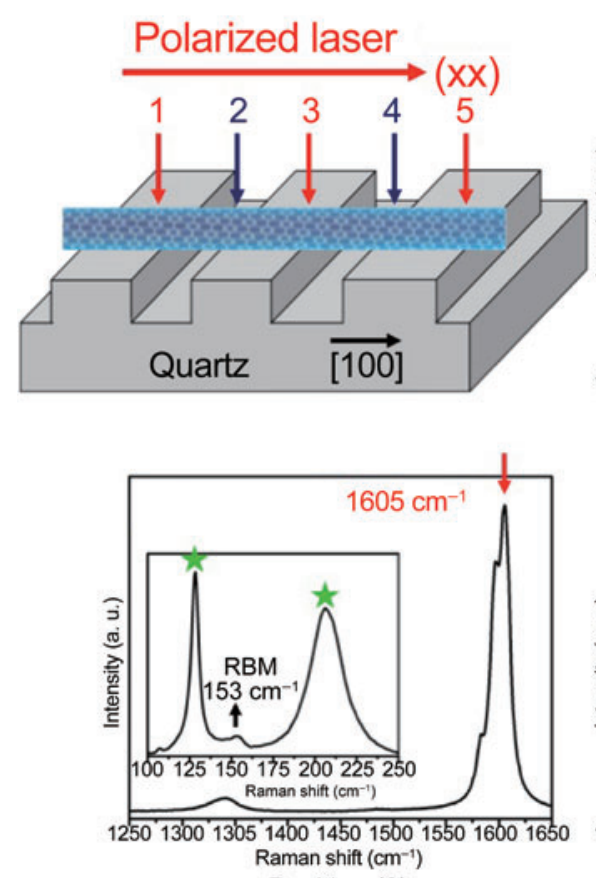

Position (3)

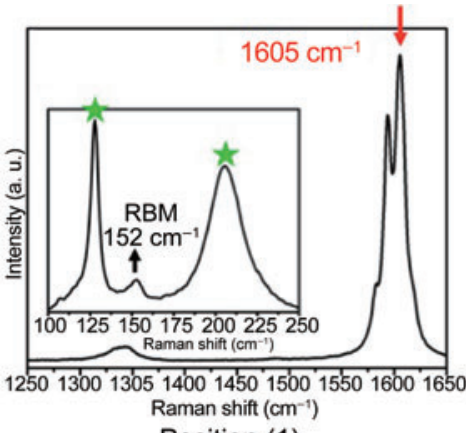

Position (1)

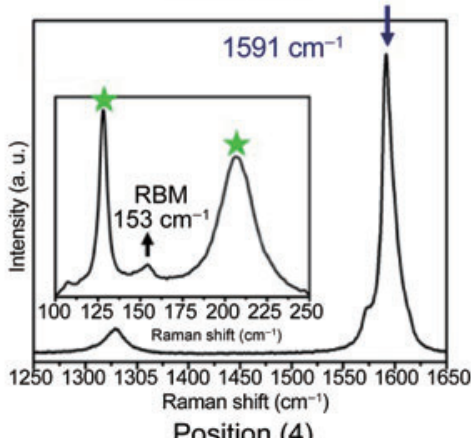

Position (4)

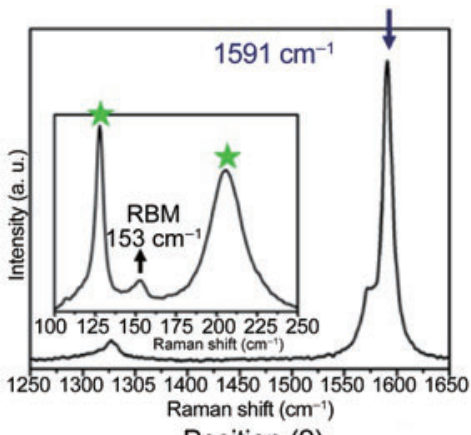

Position (2)

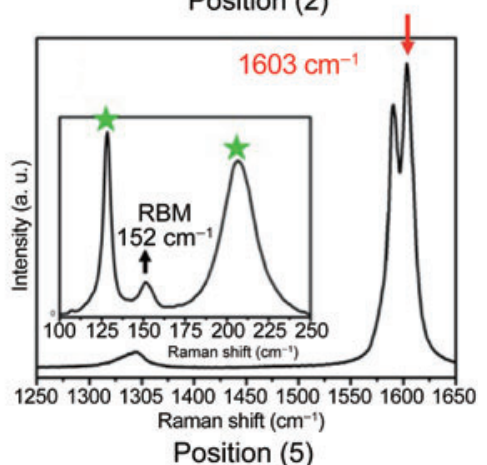

Position (5)

Figure 5 Raman spectra of the aligned SWNTs transferred onto the trenched ST-cut single crystal quartz. The top left figure is a schematic illustration of the Raman characterization. The orientation of the aligned SWNTs transferred onto the trenched quartz is parallel with the $X$ [100] direction of the lattice. Figures marked with 1 to 5 represent the Raman characterization of the sections of the nanotube in contact with the quartz (positions 1, 3, 5 in the schematic illustration) and the sections suspended over the trench (positions 2, 4 in the schematic illustration) of the same SWNT. Each of the inserted spectra depicts the RBM of the SWNT measured simultaneously with the G-band. The sharp peaks marked with green stars in the RBM spectra correspond to the Raman spectra of the quartz. The RBM and G-band frequencies are $152 \mathrm{~cm}^{-1}$ and $1605 \mathrm{~cm}^{-1}$ (position 1), $153 \mathrm{~cm}^{-1}$ and $1591 \mathrm{~cm}^{-1}$ (position 2), $153 \mathrm{~cm}^{-1}$ and $1605 \mathrm{~cm}^{-1}$ (position 3), $153 \mathrm{~cm}^{-1}$ and $1591 \mathrm{~cm}^{-1}$ (position 4), $152 \mathrm{~cm}^{-1}$ and $1603 \mathrm{~cm}^{-1}$ (position 5), respectively 
atom vibrations along the nanotube axis) and the other centered at about $1570 \mathrm{~cm}^{-1}\left(\mathrm{G}^{-}\right.$, associated with vibrations of carbon atoms along the circumferential direction) [21]. As is well know, the C-C vibrational frequency $\omega_{c-c}$ is approximately correlated to $k_{\mathrm{c}-\mathrm{c}}{ }^{0.5}$ (where $k$ is the force constant). The strong interaction between the SWNT and quartz substrate may deform the SWNT on quartz; this deformation of the SWNT results in the deformation of $\mathrm{C}-\mathrm{C}$ bonds, which may increase the value of $k_{\mathrm{c}-\mathrm{c}}$. The edge of the SWNT was structurally deformed dramatically, which may cause the obvious "up-shift" of the G-band. Also, the bottom layer of the deformed SWNT has a similar structure to monolayer graphene on this substrate. A monolayer of graphene always shows peaks in its Raman spectrum at $1615 \mathrm{~cm}^{-1}$ because of its affinity to the substrate [22]. When the SWNTs were transferred onto $\mathrm{SiO}_{x}$, the affinity force between the SWNTs and the quartz lattice disappeared. As a result, the structural deformation and the "up-shifts" of the $\mathrm{G}$ band peaks disappeared simultaneously. Further theoretical modeling is underway to investigate this hypothesis.

\section{Conclusions}

In summary, we present a CVD method for the growth of high-density, long, and uniform SWNT arrays on ST-cut single crystal quartz substrates using a high ratio of methanol:ethanol as carbon feed gas. It was evident that introducing a higher ratio of methanol:ethanol during growth can improve the density and length of the well-aligned SWNTs in the arrays. The density of these nanotubes can be as high as 20 SWNTs/ $\mu \mathrm{m}$ and the length can reach around 1 $\mathrm{mm}$. Furthermore, applying a high ratio of methanol: ethanol during growth significantly increases the interaction between the SWNTs and quartz. This is evident from the obvious "up-shifts" of the G-band frequencies of the aligned SWNTs in their Raman spectra. It is believed that the G-band "up-shift" originates from the $\mathrm{C}-\mathrm{C}$ deformation induced by the strong SWNT/quartz interaction. We believed that the results presented here represent important progress toward an understanding of the growth mechanism of aligned SWNTs on single crystal substrates. Through this increased understanding and by utilizing the ability to manipulate the affinity force between the nanotube and the substrate, it is hoped that high quality nanotubes with finely tuned characteristics can be produced for nanoelectronic applications.

Additional remark: A few days before the submission of this manuscript, we became aware of a related new publication from Prof. Shim's group in University of Illinois at Urbana-Champaign [23]. Their work shows that shifts in the Raman spectra of SWNTs grown on quartz can be attributed to differences in thermal expansion coefficient between the nanotubes and the quartz. These explanations, however, seem inconsistent with our results shown in Fig. 5 and Fig. S-2. All the SWNTs in Fig. 5 and Fig. S-2 were transferred onto the quartz surface and no cooling step was involved in the transfer process. So we are more inclined to believe that the shifts of the Raman spectra origin from the strong interaction between the nanotubes and the quartz.

\section{Acknowledgements}

The work is supported in part by a grant from Naval Research Laboratory (NRL) (N00173-04-1-G902) and Office of Naval Research (ONR) (N00014-09-1-0163). Y. L. acknowledges financial support of the National Natural Science Foundation of China (NSFC) (Nos. 50772002 and 90406018) and the Ministry of Science and Technology of China (Nos. 2006CB932403, 2007CB936202, and 2006CB932701) of China.

Electronic Supplementary Material: A representative SEM image of the marked area in Fig. 2(a) and SEM images and Raman spectra of the SWNTs transferred onto a quartz wafer with $90^{\circ}$ and $45^{\circ}$ angles along the [100] direction. Supplementary materials are available in the online version of this article at http://dx.doi. org/10.1007/s12274-009-9093-9 and are accessible free of charge.

\section{References}

[1] Kocabas, C.; Dunham, S.; Cao, Q.; Cimino, K.; Ho, X. N.; Kim, H. S.; Dawson, D.; Payne, J.; Stuenkel, M.; Zhang, 
H.; Banks, T.; Feng, M.; Rotkin, S. V.; Rogers, J. A. Highfrequency performance of submicrometer transistors that use aligned arrays of single-walled carbon nanotubes. Nano Lett. 2009, 9, 1937-1943.

[2] Kocabas, C.; Kim, H. S.; Banks, T.; Rogers, J. A.; Pesetski, A. A.; Baumgardner, J. E.; Krishnaswamy, S. V.; Zhang, $H$. Radio frequency analog electronics based on carbon nanotube transistors. Proc. Natl. Acad. Sci. U.S.A. 2008, 105, 1405-1409.

[3] Cao, Q.; Kim, H. S.; Pimparkar, N.; Kulkarni, J. P.; Wang, C. J.; Shim, M.; Roy, K.; Alam, M. A.; Rogers, J. A. Medium-scale carbon nanotube thin-film integrated circuits on flexible plastic substrates. Nature 2008, 454, 495-500.

[4] Cubukcu, E.; Degirmenci, F.; Kocabas, C.; Zimmler, M. A.; Rogers, J. A.; Capasso, F. Aligned carbon nanotubes as polarization-sensitive, molecular near-field detectors. Proc. Natl. Acad. Sci. U.S.A. 2009, 106, 2495-2499.

[5] Rao, S. G.; Huang, L.; Setyawan, W.; Hong, S. H. Largescale assembly of carbon nanotubes. Nature 2003, 425, 36-37.

[6] LeMieux, M. C.; Roberts, M.; Barman, S.; Jin, Y. W.; Kim, J. M.; Bao, Z. N. Self-sorted, aligned nanotube networks for thin-film transistors. Science 2008, 321, 101-104.

[7] Ural, A.; Li, Y. M.; Dai, H. J. Electric-field-aligned growth of single-walled carbon nanotubes on surfaces. Appl. Phys. Lett. 2002, 81, 3464-3466.

[8] Huang, S. M.; Maynor, B.; Cai, X. Y.; Liu, J. Ultralong, well-aligned single-walled carbon nanotube architectures on surfaces. Adv. Mater. 2003, 15, 1651-1655.

[9] Zhou, W. W.; Han, Z. Y.; Wang, J. Y.; Zhang, Y.; Jin, Z.; Sun, X.; Zhang, Y. W.; Yan, C. H.; Li, Y. Copper catalyzing growth of single-walled carbon nanotubes on substrates. Nano Lett. 2006, 6, 2987-2990.

[10] Ismach, A.; Segev, L.; Wachtel, E.; Joselevich, E. Atomicstep-templated formation of single wall carbon nanotube patterns. Angew. Chem. Int. Ed. 2004, 43, 6140-6143.

[11] Han, S.; Liu, X. L.; Zhou, C. W. Template-free directional growth of single-walled carbon nanotubes on a- and r-plane sapphire. J. Am. Chem. Soc. 2005, 127, 52945295.

[12] Kocabas, C.; Kang, S. J.; Ozel, T.; Shim, M.; Rogers, J. A. Improved synthesis of aligned arrays of single-walled carbon nanotubes and their implementation in thin film type transistors. J. Phys. Chem. C 2007, 111, 17879-
17886.

[13] Kang, S. J.; Kocabas, C.; Ozel, T.; Shim, M.; Pimparkar, N.; Alam, M. A.; Rotkin, S. V.; Rogers, J. A. Highperformance electronics using dense, perfectly aligned arrays of single-walled carbon nanotubes. Nat. Nanotechnol. 2007, 2, 230-236.

[14] Ding, L.; Yuan, D. N.; Liu, J. Growth of high-density parallel arrays of long single-walled carbon nanotubes on quartz substrates. J. Am. Chem. Soc. 2008, 130, 54285429.

[15] Yuan, D. N.; Ding, L.; Chu, H. B.; Feng, Y. Y.; McNicholas, T. P.; Liu, J. Horizontally aligned single-walled carbon nanotube on quartz from a large variety of metal catalysts. Nano Lett. 2008, 8, 2576-2579.

[16] Zhou, W. W.; Rutherglen, C.; Burke, P. J. Wafer scale synthesis of dense aligned arrays of single-walled carbon nanotubes. Nano Res. 2008, 1, 158-165.

[17] Ding, L.; Tselev, A.; Wang, J. Y.; Yuan, D. N.; Chu, H. B.; McNicholas, T. P.; Li, Y.; Liu, J. Selective growth of wellaligned semiconducting single-walled carbon nanotubes. Nano Lett. 2009, 9, 800-805.

[18] Kang, S. J.; Kocabas, C.; Kim, H. -S.; Cao, Q.; Meitl, M. A.; Khang, D.-Y.; Rogers, J. A. Printed multilayer superstructures of aligned single-walled carbon nanotubes for electronic applications. Nano Lett. 2007, 7, 3343-3348.

[19] McNicholas, T. P.; Ding, L.; Yuan, D. N.; Liu, J. Density enhancement of aligned single-walled carbon nanotube thin films on quartz substrates by sulfur-assisted synthesis. Nano Lett. 2009, in press, DOI: 10/1021/ nl901890x.

[20] Rutherglen, C.; Burke, P. J. Nano-electromagnetics: Circuit and electromagnetic properties of carbon nanotubes. Small 2009, 5, 884-906.

[21] Dresselhaus, M. S.; Dresselhaus, G.; Saito, R.; Jorio, A Raman spectroscopy of carbon nanotubes. Phys. Rep. 2005, 409, 47-99.

[22] Rohrl, J.; Hundhausen, M.; Emtsev, K. V.; Seyller, Th.; Graupner, R.; Ley, L. Raman spectra of epitaxial graphene on SiC(0001). Appl. Phys. Lett. 2008, 92, 201918.

[23] Ozel, T.; Abdula, D.; Hwang, E.; Shim, M. Nonuniform compressive strain in horizontally aligned single-walled carbon nanotubes grown on single crystal quartz. ACS Nano 2009, 3, 2217-2224. 